\title{
De la autodeterminación a la autocertificación del Turismo de Base Comunitaria en el Destino Siete Lagos en la Comuna de Panguipulli: Propuesta para una fundamentación desde un enfoque en derechos
}

\author{
Felipe Guerra Schleef
}

Lc. Ciencias Jurídicas y Sociales de la Universidad Austral de Chile Magister en Derecho con mención en Derecho Público de la UACh

NIAP "Economías del Sur” del CEAM-UACh.

Universidad Austral de Chile

$M^{a}$ Luisa Gómez Comi

Lc. en Derecho y Lc. en Antropología

por la Universidad Complutense de Madrid.

. Magister (c) en Derechos Humanos por la UNED.

NIAP "Economías del Sur" del CEAM-UACh.

Universidad Austral de Chile

\section{Resumen}

Este trabajo realiza un análisis crítico del contexto normativo nacional e internacional, con el propósito de ofrecer una propuesta que contribuya a la fundamentación de porqué es más adecuado que los procesos de certificación de las iniciativas de Turismo de Base Comunitaria sean desarrollados por las propias comunidades locales y pueblos indígenas, que son los actores locales y anfitriones en el Destino Siete Lagos de la Comuna de Panguipulli en la Región de Los Ríos, al sur de Chile. Para ello se realiza una revisión sistemática del actual Sistema de clasificación, calidad y seguridad de los prestadores turísticos que introduce la Ley de Turismo, que permita pasar de la certificación de la calidad y seguridad de los prestadores turísticos a la autocertificación. El derecho de los pueblos indígenas y comunidades locales de decidir sus propias prioridades en lo que atañe al proceso de desarrollo en la medida en que éste les afecte, y de controlar sus propios procesos de desarrollo económico, social y cultural, es la base conceptual y jurídica que sustenta el impulso y desarrollo de procesos de autocertificación de la calidad turística. Esto ofrece una base mínima de elementos para generar un diálogo entre autoridades, pueblos indígenas y comunidades locales, así como argumentos para el reconocimiento, fomento y promoción de dicho proceso, que va en la misma dirección de las actuales tendencias en el desarrollo de la actividad turística por parte de comunidades a nivel latinoamericano.

Palabras clave: Turismo de Base Comunitaria, autocertificación turística, autodeterminación. 


\title{
Self-certification of Community Based Tourism as a materialization of the prin- ciple of self-determination: Proposal for a foundation from a rights approach.
}

\section{Felipe Guerra Schleef}

Lc. Ciencias Jurídicas y Sociales de la Universidad Austral de Chile Magister en Derecho con mención en Derecho Público de la UACh

NIAP "Economías del Sur" del CEAM-UACh.

Universidad Austral de Chile

\author{
$M^{a}$ Luisa Gómez Comi \\ Lc. en Derecho y Lc. en Antropología \\ por la Universidad Complutense de Madrid. \\ . Magister (c) en Derechos Humanos por la UNED. \\ NIAP "Economías del Sur" del CEAM-UACh. \\ Universidad Austral de Chile
}

\begin{abstract}
This paper carries out an exhaustive review of the national and international regulatory framework in order to provide an extensive/thorough defense of why it is more appropriate that the local communities and indigenous people develop their own Certification Processes concerning the Community-Based Tourism initiatives based on "Destino Siete Lagos, Comuna de Panguipulli, Región de Los Ríos", in the south of Chile.

By carefully and thoroughly analyzing the current Classification, Quality and Safety system of Tourism Service Providers introduced by the Tourism Law, it proves that it is possible to move from the tourism service providers' quality and security certification system to a self-certification.

The right of indigenous people and local communities to decide their own priorities with regards to the development process (as far as it affects/concerns them) and to control their own processes of economic, social and cultural development is the conceptual and legal basis which supports the launch and development of self-certification processes of touristic quality. This provides a basic platform/framework to generate an open dialogue between authorities, indigenous people and local communities, as well as arguments for the recognition, promotion and acknowledgement of this process, which fits with current trends in the development of the touristic activities carried out by communities at the Latin American level.
\end{abstract}

Keywords: Community - Based Tourism, self - certification, self - determination. 


\section{Marco metodológico}

En este trabajo se realiza una reflexión crítica sobre la fundamentación jurídica de los procesos de autocertificación del Turismo de Base Comunitaria (TBC), a la luz del marco normativo nacional e internacional y desde un enfoque en derechos. Para ello, en primer lugar, efectuamos un análisis sistemático de datos cualitativos secundarios recogidos en diversos talleres, reuniones y diálogos de trabajo realizados con comunidades locales y mapuche-huilliche de la comuna de Panguipulli durante el periodo 2012-2014. Dicha información se generó en el contexto de la ejecución por parte del equipo de trabajo del Centro Transdisciplinario de Estudios Ambientales y Desarrollo Humano Sostenible de la Universidad Austral de Chile (CEAM-UACh) de sendos proyectos de desarrollo comunitario, ambos financiados por CORFO ${ }^{1}$. Se detectó que uno de los problemas ampliamente evidenciado por los actores locales, se refería a los obstáculos que presenta el Sistema de Certificación de la Calidad Turística creado por la Ley de Turismo, para que los pequeños emprendimientos del Destino Siete Lagos en la Comuna de Panguipulli puedan acceder al mismo. Esto se produce tanto por las barreras de entrada que el sistema de estandarización de la calidad turística impone a los micro-emprendimientos, como debido a que, en muchos casos, dichas barreras suponen sacrificar las particularidades propias y distintivas que caracterizan el turismo desarrollado por los pueblos indígenas y comunidades locales.

Así, en segundo lugar, procedemos a realizar un análisis crítico del contexto normativo nacional e internacional, que pueda servir para orientar y contribuir a la fundamentación de porqué es más adecuado que los procesos de certificación de las iniciativas de TBC sean desarrollados por las propias comunidades locales y pueblos indígenas, que son los actores locales y anfitriones en el Destino Siete Lagos. Para ello, realizamos una revisión sistemática del actual Sistema de clasificación, calidad y seguridad de los prestadores turísticos que introduce la Ley de Turismo desde un enfoque en derechos humanos, para sentar las bases que permitan pasar de la certificación de la calidad y seguridad de los prestadores turísticos a la autocertificación del TBC.

\footnotetext{
1 Los proyectos son: Proyecto de Transferencia Tecnológica (PDT) "Turismo de intereses especiales y desarrollo con base local en el destino siete lagos" y "Nodo de Turismo Comunitario en las zonas de amortiguación del Parque Nacional Villarrica y Reserva Nacional Mocho Choshuenco, comuna de Panguipulli". Algunos de los temas aquí expuestos son desarrollados extensamente en los informes de trabajo Hacia un proceso de autocertificación comunitaria de calidad de las iniciativas turísticas en el Destino Siete Lagos: Una perspectiva normativa global, regional, nacional y local (Guerra, 2014b) y Análisis crítico de los modelos de gestión indirecta en ASPE: Un estudio desde el Derecho Administrativo y el Derecho Internacional para la propuesta de un modelo de cogestión en el contexto del desarrollo turístico comunitario (Guerra, Gómez[col.], 2014) emanados de estos proyectos. De la misma manera, en el marco de los mismos se han realizado numerosos informes de trabajo por parte de los componentes del equipo, que constituyen un apoyo y referente para este trabajo.
} 


\section{Punto de partida: El turismo de base comunitaria como actividad diferenciada}

Enlosúltimosaños, anivelglobal, sehaidoconsolidandolaideadelTBCcomounaactividadcon potencial para mejorar las economías locales eingresos familiares, resignificar las prácticas y conocimientos tradicionales, ofreciendo también nuevas oportunidades a los actores locales para permanecer en sus territorios (Morales, 2006; Pereiro, 2006). Chile no ha sido la excepción. En el caso de la Región de los Ríos, en el sur de dicho país, el

TBC ha sido reivindicado por comunidades indígenas y rurales como un método de control territorial y fuente de subsistencia al presentarse como una propuesta de desarrollo territorial culturalmente respetuosa y económicamente viable, con el potencial de mejorar las condiciones de empleo y los ingresos familiares, siempre y cuando se desarrolle en un marco de respeto de los derechos humanos colectivos de las comunidades locales y rurales (Skewes, Henríquez y Pilquimán, 2012).

Sin embargo, el turismo no deja de ser una actividad económica, y como tal, se encuentra sometida a las relaciones propias del mercado y a las regulaciones que rigen este sector productivo. Bajo estos parámetros, en un contexto altamente competitivo y donde se privilegia la eficiencia económica, las características propias de las iniciativas desarrolladas por familias y comunidades locales no son tenidas en cuenta, pues se tiende a fomentar el turismo de masas. Esta presión termina por favorecer la informalidad de muchos emprendimientos comunitarios y amplía su brecha respecto a otros tipos de ofertas turísticas, impidiendo que se desarrollen y consoliden adecuadamente. Sin perjuicio de esto, cabe destacar que los microemprendimientos colectivos han tratado de superar estas adversidades a través de modelos de organización cooperativa, o el establecimiento de relaciones de redes o alianzas estratégicas, avanzando hacia lo que se ha denominado como encadenamientos de buenas prácticas productivas (Pacheco, Henríquez, Sampaio y Oyarzún, 2011).

En el contexto descrito, se inserta la Ley $\mathrm{N}^{\circ} 20.423$ de 2010, que crea el Sistema Institucional para el Desarrollo Turístico (Ley de Turismo), intentando ofrecer un marco regulatorio general para este sector, dada su importancia creciente y estratégica para la economía del país. Entre otras cosas, esta Ley busca mejorar la estructura normativa de la actividad turística, para aprovechar el crecimiento explosivo de su demanda a nivel mundial, la cual había estado asociada exclusivamente a lo que se conoce como "turismo de enclave o de sol y playa". Aunque la Ley reconoce la existencia de "nuevos productos turísticos" y dirige sus esfuerzos a potenciarlos, particularmente en lo que se refiere al turismo de 
intereses especiales (TIE) ${ }^{2}$, sigue operando en ella una visión fundamentalmente basada en el aprovechamiento paisajístico y los atractivos naturales lejos de toda civilización, que es, a juicio de los redactores de esta ley, "donde nuestro país tiene mucho que ofrecer" (Biblioteca del Congreso Nacional, 2010). Esto desatiende y omite cualquier referencia a las particularidades o a los aportes que han realizado al desarrollo de la actividad turística los pueblos indígenas y las comunidades locales a través del TBC. Además, esta ley crea un Sistema de Clasificación, Calidad y Seguridad de los prestadores turísticos que -al ofrece un marco normativo con un enfoque en el turismo de masas-, promueve, en aras de la calidad, la estandarización sin considerar las particularidades propias de aquellos pueblos, comunidades y territorios donde se desenvuelve la mayor parte de la actividad turística.

\section{Pluralismo jurídico: fundamentos de la autocertificación en el derecho de los pueblos indígenas a la autodeterminación.}

El Título VII de la Ley de Turismo crea un sistema de clasificación, calidad y seguridad de los prestadores turísticos que "comprende un registro de los servicios turísticos agrupados por tipo [...] y la constatación del cumplimiento de los criterios de calidad y estándares de seguridad establecidos en aquél y en las normas técnicas correspondientes" (art.30)3 . Así, quienes quieran acceder a la certificación de la calidad turística, deberán, en primer lugar, estar inscritos en el Registro Nacional de Clasificación (arts.31-32). Posteriormente, alguno de los organismos certificadores reconocidos por el Instituto Nacional de Normalización (INN), corporación de derecho privado sin fines de lucro creada por CORFO, constatará y acreditará el nivel de cumplimiento con los requisitos establecidos por las normas técnicas correspondientes que el mismo Instituto elabora con laparticipación de un sector de laindustria ${ }^{4}$. Obtenida esta certificación, se puede optar al Sello de Calidad Turística, que es entregado y administrado por Servicio Nacional de Turismo(SERNATUR), quienademásseencargadelafiscalizaciónysupervisióndelSistema.

Estamos ante un sistema normativo autónomo, donde un sector de la industria turística, con el reconocimiento de la ley, lidera e impone un proceso normativo donde el lenguaje común es el técnico-económico. Esto se manifiesta en la utilización de las lógicas propias 2 El TIE incluye el turismo aventura, ecoturismo, turismo rural, turismo cultural, agroturismo, dirigiéndose hacia una demanda más selectiva (Pacheco, Henríquez, Sampaio y Oyarzún, 2011). Está estrechamente relacionado con la naturaleza y la cultura de un territorio y se contrapone al turismo de sol y playa, el que muchas veces no considera los costos socioambientales y socioculturales que genera, ni la participación de los residentes locales. El TIE no debe confundirse con el TBC, que, aunque muy cercano a este enfoque, tiene unas características propias diferenciadas. Para una caracterización del TBC, ver: Henríquez, Pilquimán y Pino (2013). 3 El establecimiento de los procedimientos del Sistema que no estén expresamente regulados en la Ley se reenvía al desarrollo ulterior de un reglamento que deberá ser expedido por el Ministerio de Economía, Fomento y Turismo, que fue aprobado mediante el Decreto Supremo No 222 de 2011 del Ministerio de Economía, Fomento y Turismo.

4 Para profundizar en el proceso de elaboración de las normas técnicas de la calidad, véase la página del INN: http://www.inn.cl/portada/index.php 
del mercado para conseguir incentivos con miras a la ordenación de la actividad y la mejora de la oferta de los servicios turísticos, a través del cumplimiento voluntario de unos estándares de calidad que ponen el acento en la satisfacción de los clientes, excluyendo las particularidades culturales de los anfitriones. Se trata de un sistema de relaciones ju rídicas plurales, donde la ley aparece reconociendo espacios relativamente autónomos de producción normativa a sujetos no-estatales, que operan bajo una racionalidad económica (dado que lo que convoca es una actividad económica) y donde existen importantes filtros para su accesibilidad (lenguaje, racionalidad, hechos etc.), logrando representarse sólo algunos intereses. Así, utilizando las categorías de análisis de Santos (2009), podríamos señalar que el sistema de certificación de la calidad turística es un sistema normativo que agrupa elementos estatales y no estatales. Desde el interior (para los sujetos que quedan dentro del Sistema) el lenguaje común es técnico-profesional, pero accesible, participativo y, en consecuencia, consensual. No obstante, desde el exterior, para los sujetos que no logran sortear los filtros (pero que son igualmente afectados por este Sistema) prevalece un lenguaje técnico-profesional de difícil acceso, no participativo y no consensual. Así, desde el exterior, se trata de un sistema normativo donde los componentes estructurales del derecho que priman son la burocracia frente a la retórica, y la violencia simbólica ${ }^{5}$.

Por el contrario, un proceso de autocertificación, podría ser caracterizado como un mecanismo de certificación turística liderado por las comunidades, donde éstas asumen el rol de agentes responsables, participando de forma directa en el proceso de planificación y desarrollo de la calidad de sus productos y destinos turísticos. Esto permitiría recoger sus necesidades de manera pertinente y concreta, mediante la creación de instrumentos regulatorios gestionados y elaborados por la propia población residente en el destino turístico. Este punto de vista implica poner énfasis en los anfitriones, sin por eso olvidar la experiencia del visitante. En este sentido, la autocertificación turística se presentaría como una manifestación concreta de la capacidad que tienen los pueblos originarios y comunidades locales de generar sus propios órdenes normativos, como resultado del reconocimiento de su derecho a decidir sobre sus propias prioridades en

5 Sin pretender ahondar en este punto, el presente documento asume como premisa descriptiva, la existencia en las sociedades contemporáneas de una pluralidad de sistemas jurídicos y jurisdiccionales que conviven (Santos, 2009). Un ejemplo pertinente en esta línea es el desarrollo del derecho internacional los derechos humanos y el derecho económico, que cada vez le ponen más limitantes a los ordenamientos jurídicos estatales. La reciente tendencia jurisprudencial del Tribunal Constitucional en materia de derechos humanos (migración y justicia militar) lo ilustra muy bien. Progresivamente el Tribunal Constitucional ha ido incorporando referencias al sistema convencional de derechos humanos y, de esta forma, elaborando una interpretación sobre el estatus y valor jurídico de los tratados internacionales sobre derechos humanos y las decisiones de los organismos internacionales de protección, como son, entre otros, la Corte Interamericana de Derechos Humanos. Así, independiente de las concepciones y preferencias hegemónicas sobre qué ordenamiento jurídico (estatal, internacional, comunitarios etc.) debiera primar en un determinado caso, lo cierto es que en la actualidad existen diversos sistemas jurídicos interactuando, cada uno con su propia regla de reconocimiento. De esta forma, tal como señala Cover (2002), la posición estatista que considera que el Estado y su orden de jerarquías tiene asignada la capacidad exclusiva de crear derecho, "[...] confunde $[\ldots]$ el estatus de la interpretación con el estatus de la dominación política". 
lo que atañe al control de su proceso de desarrollo económico, social y cultural, en la medida en que éste afecta sus vidas, creencias, instituciones, bienestar espiritual y territorios. En otras palabras, entendemos la autocertificación como una expresión del principio de autogobierno y autodeterminación de las comunidades locales y pueblos originarios, de carácter y ejercicio colectivo tal como viene siendo consagrado en varios instrumentos internacionales sobre derechos humanos ${ }^{6}$.

\section{En el caso de los pueblos originarios, el derecho de autodeterminación} recibe una significación especifica, al ser recogido en un conjunto de normas relativas específicamente a estos pueblos, como son el Convenio 169 sobre Pueblos Indígenas y Tribales en países independientes de la Organización Internacional del Trabajo, la Declaración de las Naciones Unidas sobre los derechos de los Pueblos Indígenas y más recientemente en la Declaración americana sobre los Pueblos Indígenas ${ }^{7}$. Dichas normas configuran un consenso internacional básico en torno a un régimen de reparación y reconocimiento de los pueblos originarios como grupos humanos preexistentes,

6 Estos sujetos colectivos, titulares del derecho, se identifican como pueblos. Para un análisis en perspectiva histórica y jurídica del desarrollo de este principio internacional y derecho humano colectivo en su especifidad para los pueblos indígenas, ver Anaya (2005:135ss.).

7 El derecho a la libre determinación surgió vinculado a la idea de la independencia política de los pueblos coloniales sometidos a una potencia extranjera, entendiéndose por ello que se ejercía una sola vez, y suponía su emancipación de la metrópolis. De ahí la reticencia de muchos gobiernos nacionales a reconocer a los pueblos originarios presentes en sus territorios nacionales como pueblos, por el temor a la secesión política y fragmentación de los Estados. También por eso la matización del art. 1 del Convenio 169 sobre que no debe interpretarse pueblos "en el sentido de que tenga implicación alguna en lo que atañe a los derechos que pueda conferirse a dicho término en el derecho internacional. En este sentido, Stavenhagen (2002:184ss). Ahora bien, en el Convenio 169 el principio de autodeterminación de los pueblos originarios se desprende de varias disposiciones, entre ellas aquellas que contienen derechos de contenido político, transversales a los demás derechos que se consagran (art. 7). En la Declaración se hace referencia explícita al derecho de autodeterminación en términos de libre determinación (art.3), en condiciones similares al art. $1^{\circ}$ común de los Pactos Internacionales de Naciones Unidas sobre Derechos Civiles y Políticos, y Derechos Económicos, Sociales y Culturales (Aylwin, 2009:17). Esta interpretación ha sido mantenida por la Corte Interamericana de Derechos Humanos (2007) en el caso del Pueblo Saramaka vs. Surinam.

El artículo 4 de la Declaración precisa el contenido del derecho a la libre determinación, que implica los derechos " [...] a la autonomía o al autogobierno en las cuestiones relacionadas con sus asuntos internos y locales, así como a disponer de medios para financiar sus funciones autónomas". De esta forma, el derecho de autodeterminación de los pueblos indígenas corresponde al autogobierno y autogestión de los recursos propios, en virtud de lo cual pueden decidir autónomamente las prioridades de su propio desarrollo económico, social y cultural, como así también de disponer libremente de sus riquezas y recursos naturales, no pudiendo ser privados de sus propios medios de subsistencia (Yáñez, 2006:501). Esto implica el control interno, político y económico de los territorios, medio ambiente, conocimientos tradicionales, expresiones culturales, desarrollo económico, etc. De este modo, entendemos que la libre determinación de los pueblos los originarios adquiere toda una nueva gama de connotaciones respecto a aquella con la que surgió, siendo el hecho de que su ejercicio es reiterado y se refiere a la libre determinación de sus asuntos propios y control de su desarrollo entendido en sentido amplio. Además, debe ser la inspiración de cualquier proceso de autorregulación y autocertificación de la actividad turística, al estar comprometido en ella el desarrollo económico, así como la cultura y territorios de estos pueblos. 
culturalmente diferenciados y que han sufrido desigualdades dentro de los Estados en los que viven, en razón a los procesos históricos de conquista, colonización y formación de los Estados Nacionales en sus territorios (Anaya, 2005; Aylwin, Meza-Lopehandía, Yáñez, 2014).

En el caso de la comuna de Panguipulli, lo señalado precedentemente resulta fundamental, al corresponder íntegramente su área geográfica-administrativa al territorio ancestral mapuche-huilliche y donde el $35,37 \%$ de su población se auto-identifica como mapuche ${ }^{8}$.

Esto hace pertinente situarnos desde el derecho de los pueblos originarios a decidir sus propias prioridades en su proceso de desarrollo, a la hora de evaluar, diseñar, fomentar y vigilar una política pública de la actividad turística en dicho territorio. Además, lo anterior implica tomar distancia de una justificación del proceso de autocertificación sustentado en aspectos puramente ambientales o concepciones estáticas sobre la cultura de los pueblos originarios y comunidades locales, que tienda a la folcklorizacion de la vida campesina y mapuche. Así, si entendemos que la especificidad de los derechos reconocidos a los pueblos originarios y comunidades locales tiene su fundamento en su autoreconocimiento como pueblos (que como tal tienen derecho al autogobierno), introducimos un componente propiamente político y jurídico, que les restituye la posibilidad de deliberar y disponer libremente sobre el proceso de desarrollo económico o cualquier materia que les incumba directamente. Esto se traduce necesariamente en la capacidad de generación de órdenes normativos autónomos y propios, respaldado en el derecho internacional de los derechos humanos y en la igualdad de trato que merecen las personas que integran estos pueblos y comunidades, como seres responsables para asumir el control de susvidasdentrodelmarcodelordenamientojurídicoestataleinternacional(Anaya,2005:141).

8 Reportes Estadísticos Comunales 2012, Biblioteca del Congreso Nacional de Chile. Disponible en: http:// reportescomunales.bcn.cl/2012/index.php/Panguipulli\#Poblaci.C3.B3n_seg.C3.BAn_etnia_declarada_Casen_2003-2006-2009

9 Fue promulgado mediante el Decreto Supremo N ${ }^{\circ} 236$ de 2008, del Ministerio de Relaciones Exteriores, y entró en vigor el 15 de septiembre de 2009. Es un tratado internacional de derechos humanos, lo que significa que tiene un estatus y valor jurídico especial con respecto a nuestro ordenamiento jurídico interno, tal como lo prescribe el art.5 inciso $2^{\circ}$ de la Constitución Política de la República de Chile. En efecto, los derechos reconocidos en tratados internacionales sobre derechos humanos ratificados y que se encuentren vigentes son, junto a los demás derechos garantizados por la Constitución, un límite al ejercicio de la soberanía, siendo imperativo para los órganos del Estado respetar y promover tales derechos. Así lo ha señalado el Tribunal Constitucional (2014). 
IV. El Convenio 169 de la OIT y las Declaraciones sobre Turismo comunitario de la Red de Turismo Sostenible en América Latina impulsada por la OIT. Implicancias en el proceso de autorregulación y gestión comunitaria de la actividad turística.

\section{El Convenio 169 de la OIT}

El Convenio 169 de la OIT $^{9}$ consagra derechos referidos exclusivamente a los pueblos originarios en su condición de sujetos colectivos, lo que también implica el reconocimiento de su ejercicio de forma colectiva. El fundamento para ello se encuentra contenido en el Preámbulo, y debe ser interpretado desde la idea de autodeterminación consagrada en la parte introductoria. En su articulado, este Convenio se refiere a una amplia gama de derechos en diversas materias (tierra y territorios, acceso a los recursos naturales, política, salud, educación, formación profesional, condiciones de empleo, contactos a través de las fronteras etc), siendo los de carácter político (consulta, participación y al consentimiento previo libre e informado) la "piedra angular" de este tratado ${ }^{10}$. Por el tema que nos convoca, nos concentraremos en los derechos de carácter económico, los que de todas maneras se encuentran cruzados en sus fundamentos por elementos políticos, territoriales y culturales.

En efecto, el Convenio 169 considera una serie de derechos que debieran orientar la actuación de los órganos de la Administración del Estado que toman decisiones que inciden sobre las iniciativas turísticas desarrolladas por comunidades y familias indígenas, al afectar sus posibilidades de desarrollo, territorios, cultura, modos de vida e instituciones tradicionales. En este sentido, no hay que olvidar que el turismo puede ser una actividad provechosa para las comunidades locales al generar efectos beneficiosos, pero, también puede ser invasiva y afectar al entorno social, cultural y natural donde se desenvuelve. De esta forma, un proceso de autocertificación de las actividades y servicios turísticos, que surja de las propias comunidades y sea reconocido por la Administración del Estado, podría contribuir a que los pueblos originarios transformen el turismo en una actividad complementaria, que mejore su situación material y económica, aspirando a eliminar las diferencias socioeconómicas que existen entre los miembros indígenas y el resto de la comunidad nacional de una manera compatible con sus aspiraciones y formas de vida (art. 2 del Convenio 169 de la OIT).

10 Así lo afirmó la Comisión de Expertos en Aplicación de Convenios y Recomendaciones, en su observación general sobre Pueblos Indígenas y Tribales del año 2008 (Oficina Internacional del Trabajo, 2009). Para un desarrollo sobre este tema en la doctrina nacional ver Aylwin, Meza-Lopehandía, Yáñez (2014). 
Las particulares circunstancias geográficas, económicas y exigencias que la regulación estatal le impone a las iniciativas turísticas rurales, favorecen que el turismo desarrollado y gestionado por comunidades indígenas y rurales se desenvuelva en un plano de desigualdad, dado que estos elementos contingentes impiden cumplir con muchas de las exigencias requeridas para formalizar las iniciativas turísticas en zonas rurales. Así, el propio diseño de los estándares normativos, en los hechos sostiene la informalidad y, en consecuencia, termina por generar diferencias arbitrarias que podrían ser perfectamente corregidas si se tomaran en atención las particulares y aspiraciones de los emprendimiento comunitarios y particulares de las iniciativas turísticas rurales. Desde esta lógica, resultan del todo oportuno iniciativas de fomento turístico pertinentes a la realidad rural y cultura indígena y campesina como el Programa de Turismo Rural de INDAP.

En esta línea, también se prevé la posibilidad de adoptar medidas especiales (con el consentimiento de los pueblos interesados) de acuerdo con el art. $4^{\circ}$ del Convenio 169 de la OIT. Esto, con el objeto de proteger las personas, instituciones, los modos de vida, economía, trabajo, cultura $\mathrm{etc}^{11}$. En derecho comparado, esto se conoce como acciones afirmativas, que resultan fundamentales para superar circunstancias de desigualdad temporal y eventuales (Aylwin, Meza-Lopehandía, Yáñez, 20014:368ss). Ahora bien, dichas medidas excepcionales no constituyen un privilegio a las personas y pueblos originarios, sino un medio efectivo corregir desigualdades y preservar sus comunidades y desarrollo, que debiera ser entendido desde el derecho a participar con igualdad de oportunidades en la vida nacional ${ }^{12}$.

Por otra parte, de acuerdo con el art. 5 del Convenio 169 de la OIT, los estados tienen el deber de reconocer y proteger los valores y prácticas sociales, culturales, religiosas y espirituales propias de los pueblos originarios, lo que supone que tienen que ser debidamente tomadas en consideración la índole de los problemas que se plantean a estos pueblos tanto colectiva como individualmente. El mismo artículo agrega que "deberá respetarse la integridad de los valores, prácticas e instituciones de esos pueblos", además del deber de adoptar, con la participación y cooperación de los pueblos interesados, medidas encaminadas a allanar las dificultades que experimenten al afrontar nuevas condiciones de vida y de trabajo. Este artículo, entre otros, es expresión del carácter dinámico que se le atribuye a las culturas de los pueblos originarios. En efecto, la cultura surge de aque-

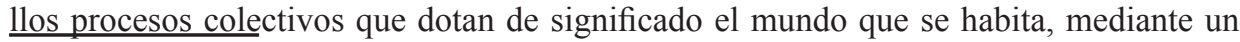
11 Un pronunciamiento judicial en este sentido en autos caratulados Millaray Huichalaf Pradines y Otros C/ Juan H. Ortiz, donde la Corte de Apelaciones de Valdivia (2012) toma como referencia el artículo 4 del Convenio 169 para fundamentar la idoneidad del recurso de protección a la hora de tutelar los derechos colectivos de los pueblos originarios.

12 En este sentido ver Tribunal Constitucional de Chile (2013). En el mismo sentido, Tribunal Constitucional de Chile (2008) en sentencia de 3 de abril de 2008, sobre control de constitucionalidad de los artículos $6^{\circ}$ y $7^{\circ}$ del Convenio 169 de la OIT. 
simultáneo involucramiento y desentendimiento, identificación y objetivación. Las condiciones de vida y trabajo al cambiar tensan y dinamizan las transformaciones culturales, resinificándolas permanentemente.

Así mismo, en su art. 7, el Convenio 169 de la OIT consagra tres derechos de orden económico y político fundamentales para el TBC: 1) el derecho a decidir sus propias prioridades en lo que atañe al proceso de desarrollo, en la medida en que éste afecte a sus vidas, creencias, instituciones y bienestar espiritual y a las tierras que ocupan o utilizan de alguna manera; 2 ) el derecho de controlar su propio desarrollo económico, social y cultural y 3) el derecho a participar en la formulación, aplicación y evaluación de los planes y programas de desarrollo nacional y regional susceptibles de afectarles directamente.

El Convenio 169 de la OIT, también se establecen pautas para un pluralismo jurídico respetuoso de los derechos humanos, que debieran orientar la relación de los estados con los pueblos originarios, al reconocer el derecho de estos pueblos a conservar sus costumbres e instituciones propias, siempre que éstas sean incompatibles con los derechos humanos reconocidos en el sistema jurídico nacional e internacional (art.8). De esta forma, el Convenio eleva el reconocimiento del derecho consuetudinario de los pueblos originarios, estableciendo el deber de los Estados de tomar en consideración las costumbres de estos pueblos a la hora de aplicarles la legislación nacional y el deber de establecer procedimientos para solucionar los conflictos que puedan surgir en la aplicación de este principio.

Finalmente, el Convenio reconoce las actividades tradicionales y relacionadas con la economía de subsistencia de los pueblos originarios (como la artesanía, las industrias rurales y comunitarias, la caza, la pesca, la recolección) como factores importantes del mantenimiento de su cultura y de su autosuficiencia y desarrollo económicos, debiendo los gobiernos, con la participación de los pueblos originarios, velar para que se fortalezcan y fomenten dichas actividades. A petición de los pueblos interesados, debe facilitarse una asistencia técnica y financiera apropiada que tenga en cuenta las técnicas tradicionales y sus características culturales y la importancia de un desarrollo sostenido y equitativo.

Todos estos derechos de los pueblos originarios, de implicancias económicas, políticas y de ejercicio colectivo, deben ser considerados como un marco básico desde el cual construir y fundamentar un proceso de autocertificación de la calidad turística y de autorregulación. De esta manera, lo determinante de los derechos garantizados en el Convenio 169 de la OIT, son las obligaciones que impone a los estados en su orientación al trato que deben dar a los pueblos originarios en lo que dice relación con sus territorios, culturas, instituciones y en el proceso de desarrollo cuando este les afecte directamente, como es el caso del crecimiento y fomento de la actividad turística en la comuna de Panguipulli, territorio ancestral mapuche-huilliche. 


\section{Las Declaraciones sobre Turismo Comunitario y la Red de Turismo Sostenible (REDTURS) en América Latina impulsada por la OIT}

Prueba de que el turismo ha devenido en una actividad complementaria de las económicas locales, de promoción cultural y de resguardo territorial para los pueblos originarios y comunidades locales, ha sido el trabajo desarrollado en el marco del Convenio 169 de la OIT, fundamento jurídico bajo el cual la OIT, junto a estados, organizaciones, pueblos originarios y comunidades locales de diversos países latinoamericanos, han comenzado a trabajar en la elaboración de modelos de autogestión del turismo socialmente responsables. Fruto de estas iniciativas han sido la Declaración de Otavalo sobre Turismo Comunitario, Sostenible, Competitivo y con Identidad Cultural (Ecuador, 2001) ${ }^{13}$, la Declaración de Oaxaca (Mexica, 2002) $)^{14}$, la Declaración de San José sobre Turismo Rural Comunitario (Costa Rica, 2003) ${ }^{15}$, y la Declaración de Fortaleza $(\text { Brasil, 2008) })^{16}$. En estos instrumentos se recoge la visión, principios y aspiraciones que inspiran las prácticas de las iniciativas de TBC en el ámbito regional latinoamericano y son referentes pioneros a nivel mundial de iniciativas de turismo comunitario.

La "Red de Turismo Sostenible" (REDTURS) ${ }^{17}$ surge en 2001 como respuesta a las tendencias del mercado y a las aspiraciones formuladas por numerosas organizaciones indígenas y campesinas, y asume desde el año 2004 el papel de secretaría técnica de una red de desarrollo sostenible, con el fin de intercambiar información, difundir experiencias de

13 Fue el resultado del Encuentro Andino en Otavalo (2001), cuyo propósito era compartir los resultados de varios talleres que se habían llevado a cabo en distintos países de la región, en los que se discutió sobre las condiciones necesarias para el desarrollo de un turismo sostenible desde la perspectiva de las comunidades indígenas, y las relaciones que éstas esperaban establecer con el Estado y el sector privado turístico. El evento congregó a representantes de los gobiernos nacionales y locales, las comunidades y organizaciones indígenas, y ONGs.

14 Fue resultado de una iniciativa global de la "Indigenous Tourism Rights International" (ITRI), quien organizó el Foro Internacional de Turismo Indígena, y supuso una llamada a los grupos indígenas para "reforzar estrategias, coordinar la cooperación internacional y regional, y aumentar la participación indígena en el ecoturismo" (Pereiro, 2014).

15 Surgió del encuentro celebrado en San José de Costa Rica (2003), en el que participaron representantes de organizaciones comunitarias y cooperativas turísticas de varios países latinoamericanos con un doble propósito: debatir y concertar estrategias de promoción, mercadeo y comercialización de productos turísticos comunitarios; y deliberar y proponer directrices para establecer códigos de conducta, con el objeto de preservar la identidad cultural y la autenticidad de los destinos turísticos comunitarios (estas áreas eran las que evidenciaban mayor debilidad o amenazas como consecuencia de las relaciones de las comunidades con los operadores turísticos).

16 En ella "se afirma la idea de un turismo solidario de base comunitaria frente al convencional o de masas [...] la necesidad de un consumo turístico ético y solidario que contribuya a superar las desigualdades sociales [...] y solicita medidas legales que favorezcan estructuralmente el turismo comunitario" (Pereiro, 2014:16). 
promoción y mercadeo, compartir conocimientos legales y gerenciales, y acceder a servicios de desarrollo empresarial. Sus instrumentos fundacionales son las ya mencionadas Declaraciones de Otavalo y San José, sobre las que nos detendremos ahora brevemente.

Como destaca Maldonado (2006:59), la Declaración de Otavalo aborda tres temas fundamentales para el desarrollo del TBC en la región: la política pública de fomento del turismo comunitario, las estrategias para su aplicación y los medios para garantizar resultados concretos. En lo que dice relación con las políticas públicas, se insta a los gobiernos a formular políticas cuyo objetivo sea alcanzar un turismo sostenible, competitivo y con identidad cultural. Para esto se propone una serie de estrategias para alcanzar dicho objetivo, entre las que destaca el fomento de procesos educativos fundados en la valoración de la diversidad cultural y la equidad social, la institucionalización de una política nacional representativa, dialógica y concertada entre Estado, sector comunitario y sector privado, y la entrega de incentivos públicos que potencien el turismo comunitario como actividad económica y que minimice sus efectos nocivos en el entorno natural y cultural $)^{18}$. Todo lo anterior, en el marco del ejercicio de los derechos colectivos de los pueblos indígenas y sus comunidades, consagrados por el Convenio 169.

Por su parte, la Declaración de San José ratifica los principios y recomendaciones de la de Otavalo, definiendo además conceptos claves sobre el desarrollo de la actividad turística gestionada por comunidades ${ }^{19}$, avanzando así en el posicionamiento del TBC como un componente estratégico del desarrollo local, nacional y regional, poniendo de relieve su contribución a la generación de ingresos y empleo para el bienestar de las comunidades anfitriona (Maldonado, 2006). Por otra parte, la Declaración considera que tanto el protagonismo de las comunidades como la autogestión de los proyectos turísticos, se sustentan en los derechos colectivos de los pueblos originarios consagrados en los instrumentos internacionales analizados. Además, se re onoce que el TBC es una estrategia 17 REDTURS es una red de comunidades, instituciones de apoyo y recursos humanos que promueve una visión del desarrollo sostenible del turismo que busca compatibilizar los objetivos de eficiencia económica con los principios de equidad social, identidad cultural y preservación de los recursos naturales. Brinda apoyo a las comunidades en los procesos de formación y fortalecimiento de las redes de TBC de América Latina, para diversificar sus fuentes de empleo e ingreso, valorar su cultura y afianzar la cohesión social, acompañándolas en los procesos de reflexión, búsqueda de soluciones y aplicación de estrategias que les permitan competir con ventajas en el mercado. Seis destinos en nuestro país pertenecen a REDTURS, entre los cuales cabe mencionar la Red de Parques Comunitarios Mapu Lahual (X Región de los Lagos).

18 Entre los medios que se proponen en la Declaración para desarrollar la política y estrategias propuesta, podemos señalar: 1) alianzas institucionales entre las comunidades y sus organizaciones con gobiernos centrales y locales, la empresa privada y las ONG con el fin de concebir, poner en marcha y evaluar programas de interés común; 2) programas de capacitación técnica para profesionalizar el recurso humano comunitario, optimizar la gestión empresarial y la calidad de los servicios; 3 ) acceso de las comunidades a mercados, recursos financieros e infraestructura con fines turísticos; 4) concertar y aplicar un marco jurídico que reconozca, regule y garantice el ejercicio de la empresa turística comunitaria en los diferentes países; 5) promover las expresiones genuinas de la identidad cultural de los pueblos indígenas, fuente de la diferenciación y competitividad de sus productos turísticos. 
adecuada para garantizar mayor poder de decisión y de control sobre sus territorios, su cultura y los procesos de desarrollo en marcha (art. 7) ${ }^{20}$.

Por último, quisiéramos realizar una breve reseña sobre los códigos de conducta en el TBC, instrumentos que no deben confundirse con los procesos de autocertificación ${ }^{21}$. Existen tantos tipos de códigos de conducta como actores de los que emanan y objetivos que persiguen. En ellos se contienen normas y principios de adscripción voluntaria, que buscan propiciar un entorno adecuado para el desarrollo del turismo, maximizando los beneficios para los actores involucrados y minimizando los riesgos de esta actividad ambivalente, aunque precisamente esa voluntariedad constituye una sus principales limitaciones. La escasa participación inicial de las comunidades locales en su elaboración derivó en la inadecuación de muchos de los códigos que existían en el ámbito del turismo para las especificidades del TBC y, por tanto, en su desconocimiento e inaplicación por parte de los operadores turísticos comunitarios (Maldonado, 2006:45ss.). Para hacer frente a esa situación, en el encuentro regional que dio lugar a la Declaración de San José, se examinaron varios de estos codigos para debatir cuáles podían ser los principios y normas atingentes al $\mathrm{TBC}^{22}$. Finalmente, en esa ocasión no se consiguió consensuar una propuesta de directrices para elaborar un código de conducta desde el punto de vista del TBC, por lo que el debate se retomó en el IV Encuentro Latinoamericano, realizado en Panamá durante el año 2005, que versó expresamente sobre este tema y en cuyos resultados aún se sigue trabajando.

19 Para que un determinado proyecto turístico sea considerado de carácter comunitario, la empresa puede adoptar una de las siguientes modalidades de propiedad de los activos: comunitaria, cooperativa o asociación de proveedores finales de servicios al turista. Además, debe responder efectivamente a los intereses de la comunidad, gracias a su participación directa en el proceso de formulación y diseño del proyecto (concepción); la prestación de los servicios turísticos (operación); conducción de la empresa (autogestión y capacidad de decisión); y percepción de beneficios económicos y no económicos (ingresos, empleo, valoración cultural, preservación de territorios, fortalecimiento de la cohesión social, etc.). Sin perjuicio de esto, como señala Maldonado (2006) "[u]n negocio de carácter familiar puede también formar parte de la red, siempre y cuando goce del respaldo de la comunidad y ésta se beneficie de alguna manera".

20 En este sentido, la Declaración señala que las comunidades "[r]eafirmamos el derecho de propiedad y control de nuestras tierras y territorios -fuente de subsistencia, identidad y espiritualidad-, derecho consagrado en el Convenio núm. 169 de la OIT [...] [d] eclinamos vender o ceder en concesión nuestras tierras a personas que no sean de nuestras comunidades [...]" y también "[r]eafirmamos nuestro derecho de consulta previa y participación bien informada en los procesos de adopción de decisiones relacionados con la planificación, ejecución y evaluación de políticas y programas en materia [...] turística, en la medida en que esas decisiones afecten directamente nuestras vidas, instituciones y bienestar espiritual", la que "[...] habrá de llevarse a cabo [...] respetando las instancias y los procedimientos apropiados".

21 Maldonado (2006:50) los define como instrumentos de gestión y regulación que persiguen avanzar en la transparencia y la vigilancia de las conductas de todos los actores involucrados en el fenómeno turístico, de manera que se garantice el respeto mutuo y una convivencia intercultural enriquecedora para todos. Con su aplicación se pueden prevenir conflictos, evitar el deterioro del patrimonio comunitario, y generar beneficios económicos. 
retomó en el IV Encuentro Latinoamericano, realizado en Panamá durante el año 2005, que versó expresamente sobre este tema y en cuyos resultados aún se sigue trabajando ${ }^{23}$.

\section{El Convenio de las Naciones Unidas sobre la Diversidad Biológica y el Protocolo de Nagoya: Aportes para el proceso de autocertificación del TBC}

\section{El Convenio de las Naciones Unidas sobre la Diversidad Biológica}

De la Conferencia de las Naciones Unidas sobre el Medio Ambiente y el Desarrollo, realizada en Río de Janeiro durante el año 1992, emanaron cinco documentos internacionales: el Convenio sobre la Diversidad Biológica (CDB), la Declaración de Río de Janeiro, un documento base de la Convención de Naciones Unidas sobre Cambio Climático, la Declaración de Principios para un Consenso Mundial respecto de la Ordenación, la Conservación y el Desarrollo Sostenible relativo a los Bosques y la Agenda 21. Estos instrumentos son sumamente relevantes en el proceso de reconocimiento del plano colectivo de los derechos de los pueblos originarios y las comunidades locales $^{24}$. Por las implicaciones que tiene el medio ambiente para el TBC, resulta particularmenteinteresanteanalizarelCDB, porloqueahoranosdetendremosenestetratado ${ }^{25}$.

El Preámbulo del CDB se hace una referencia explicita a la estrecha relación que tiene la diversidad natural para el turismo, al señalar que las partes son conscientes del "valor recreativo" de la diversidad biológica y sus componentes. Además, el Preámbulo de la CDB se reconoce " $[\ldots]$ la estrecha y tradicional dependencia de muchas comunidades locales y poblaciones indígenas que tienen sistemas vida tradicionales basados en los recursos biológicos, y la conveniencia de compartir equitativamente los beneficios que se derivan de la utilización de los conocimientos tradicionales, las innovaciones y las prácticas pertinentes para la conservación de la diversidad biológica y la utilización sostenible de sus componentes".

22 Se debatieron principalmente varias experiencias comunitarias y el Código Ético Mundial de la de OMT (CEM). Sobre éste, se reconoció su pertinencia (siempre y cuando sirviera a los intereses de la comunidad) y su utilidad práctica (en la medida que regulase una conducta de los visitantes que contribuyera a la preservación de los recursos naturales y el acervo cultural y social comunitario). Una referencia a los Códigos éticos quedó plasmada en el art. 6 de la Declaración.

23 Para profundizar en el tema puede consultarse las Memoria del IV Encuentro Latinoamericano, Códigos de conducta y uso de marcas, realizado en Panamá, noviembre 2005. En Oficina Internacional del Trabajo (2006). Las directrices emanadas de los cinco grupos de trabajo pueden consultarse en Maldonado (2006:63$70)$.

24 En este sentido, para profundizar en el tema véase Yáñez (2006) y Aylwin y Cuadra (2011).

25 En la actualidad, 191 países lo han ratificado, entre ellos Chile mediante el Decreto Supremo $\mathrm{N}^{\circ} 1.963$ del Ministerio de Relaciones Exteriores en 1994. 
En este sentido, en el marco del art. 8 del CDB, que desarrolla los compromisos y obligaciones en relación con la conservación in situ, su letra j) establece el deber de los Estados Partes de respetar, preservar, mantener y fomentar las prácticas y conocimientos de los pueblos originarios y comunidades locales que entrañen estilos tradicionales de vida pertinentes para la conservación y la utilización sostenible de la diversidad biológica. Por su parte, el art. 10 c), se refiere a la utilización sostenible de los componentes de la diversidad biológica, debiendo los Estados Contratantes proteger y alentar la utilización consuetudinaria de los recursos biológicos, de conformidad con las prácticas culturales tradicionales que sean compatibles con las exigencias de la conservación o su utilización sostenible.

De esta manera, este Convenio se refirió por primera vez a la dependencia que mantiene los pueblos originarios y las comunidades locales con sus territorios y los recursos naturales presentes en ellos, así como al valor de los conocimientos tradicionales relacionados con estos recursos y la necesidad de establecer medidas para preservarlos ${ }^{26}$.

También se reafirma el importante papel que estos pueblos y comunidades han desempeñadoenlaconservaciónyordenacióndelmedioambienteyeldesarrollosustentable ${ }^{27}$.

El CDB es el único instrumento internacional en el que se abordan de manera exhaustiva cuestiones relativas a la diversidad biológica, vinculando a ésta con los pueblos originarios y las comunidades locales. A través de tres sus objetivos principales mencionados en su art.1 (la conservación de la biodiversidad, la utilización sostenible de sus componentes y la participación justa y equitativa en los beneficios que se deriven del uso de los recursos genéticos), el CDB reconoce importantes derechos a los pueblos originarios y comunidades locales, y se imponen obligaciones a los Estados Parte, pues se entiende que son "responsables de la conservación de su diversidad biológica y de la utilización sostenible de sus recursos biológicos". Para ello, el CDB le exige a los Estados Partes planificar y aplicar medidas adecuadas que ordenen las actividades humanas que puedan incidir en la reducción de la biodiversidad (o en su preservación) en los términos recogidos en el tratado. Esto es, garantizando "la participación plena y efectiva de las comunidades indígenas y locales en todas las etapas y niveles de su aplicación ${ }^{28}$ ".

26 Para profundizar en el tema, véase también, Yáñez (2006) y Aylwin y Cuadra (2011:55ss).

27 En este sentido, cabe mencionar el trabajo y deliberaciones que desde la década de los 60 ha desarrollado la Organización Internacional Unión Internacional para la Conservación de la Naturaleza (UICN), sobre el reconocimiento del importante papel de los pueblos indígenas y las comunidades locales en la conservación del medio ambiente y en el desarrollo sustentable.

28 Este es el objetivo principal del Programa de Trabajo que originó el artículo 8 j) del Convenio. Sobre su contenido específico, véase la siguiente nota a pie de página. Implica, entre otras cosas, la obligación de "desarrollar capacidades científicas, técnicas e institucionales" entre los actores directamente involucrados. 
Como se puede apreciar, esta concepción de la conservación de la biodiversidad (directamente vinculada con las actividades humanas que inciden en ella y con todos los actores involucrados de una u otra manera) eleva su ámbito a un plano propiamente político, en el cual las soluciones a las problemas que se susciten han de pasar necesariamente por el impulso de acciones coordinadas, que sean fruto de decisiones políticas e institucionales. De esta manera, en cumplimento con las obligaciones asumidas por el Estado a través del CDB y los demás documentos que éste ha originado ${ }^{29}$, los servicios públicos de la Comuna de Panguipulli no sólo han de incorporar activamente a las comunidades locales y pueblos originarios en todos procesos de planificación de políticas y demás cuestiones relativas a la toma de decisiones sobre el desarrollo de actividades que inciden en la conservación y utilización sostenible de los recursos biológicos, como es el turismo. También es fundamental que fomenten el fortalecimiento de sus capacidades para el diseño participativo de instituciones que regulen el acceso, uso y gestión de sus recursos biológicos y los conocimientos tradicionales vinculados a los mismos.

\section{Protocolo al Convenio sobre Diversidad Biológica sobre Acceso a los Recursos Genéticos y Participación Justa y Equitativa en los Beneficios que se Deriven de su Utilización}

Protocolo al Convenio sobre Diversidad Biológica sobre Acceso a los Recursos Genéticos y Participación Justa y Equitativa en los Beneficios que se Deriven de su Utilización, también conocido como el Protocolo de Nagoya ${ }^{30}$, surge con el propósito de profundizar en la regulación sobre la participación justa y equitativa en 29 Entre ellos cabe destacar El Protocolo de Nagoya (al que nos referimos más adelante) y un Programa de Trabajo compuesto por varios Elementos que agrupan tareas concretas para los Estados Parte. El Elemento 1 (Mecanismos de Participativos para Comunidades Indígenas y Locales) establece que éstos "deben adoptar medidas dirigidas a mejorar e intensificar la capacidad de las comunidades indígenas y locales para participar eficazmente en la adopción de decisiones relacionadas con el empleo de sus conocimientos, innovaciones y prácticas tradicionales de importancia para la conservación y utilización sostenible de la diversidad biológica, con sujeción a su consentimiento fundamentado previo y participación activa" (Tarea 1). Por su parte, la Tarea 2 insta a que esa participación efectiva sea a nivel internacional, regional, subregional, nacional y local, incluyendo "el acceso a los beneficios y a su distribución y la designación y ordenación de zonas protegidas". Por último, la Tarea 4 incorpora el enfoque de género, refiriéndose expresamente a la "participación plena, activa y eficaz de las mujeres en todos los elementos del programa de trabajo". Por otro lado, la Tarea 7 (Elemento 4) establece que sobre la base de las tareas mencionadas se preparen directrices para la elaboración de instrumentos que garanticen la distribución justa y equitativa de los beneficios que derivan del uso y la aplicación de los conocimientos, innovaciones y prácticas, y que las instituciones privadas y públicas interesadas en el uso de esos conocimientos, innovaciones y prácticas obtengan el consentimiento fundamentado previo de las comunidades indígenas y locales.

30 En la Cumbre Mundial sobre Desarrollo Sostenible, realizada en Johannesburgo durante el año 2002 , se realizó una convocatoria para negociar dentro del marco del CDB, un instrumento internacional que promoviera y salvaguardara la participación justa y equitativa en los beneficios derivados de la utilización de recursos genéticos. En 2004 se mandató a un Grupo de Trabajo para que elaborar y negociar un régimen internacional para aplicar efectivamente los artículos 15 y 8 j) del CDB. Tras seis años de negociaciones se adoptó el año 2010 el Protocolo de Nagoya, el que entró en vigor el 12 de octubre 2014, tras su ratificación por 51 partes del CDB. 
los beneficios que se deriven de la utilización de los recursos genéticos y el acceso a los mismos, en la línea definida por el $\mathrm{CDB}^{31}$. Chile se adhirió a este tratado en 2010, pero aún no lo ha ratificado y por ende, carece de vigencia. No obstante, tanto por el compromiso asumido por el Estado de Chile de ratificar este instrumento, consideramos interesante comentar los efectos que se derivan de este Protocolo para el TBC.

Las obligaciones que impone el Protocolo de Nagoya deben ser tenidas en cuenta en Chile, y es conveniente que las comunidades locales y pueblos originarios tengan presentes los derechos que se les reconocen. En él se desarrollan una serie de temas relevantes para el establecimiento de un marco jurídico que permita diseñar un proceso de autocertificación respetuoso con los derechos humanos y directrices internacionales, en los territorios donde se está trabajando. Así, de su articulado destacamos el derecho a la participación sin discriminación en los procesos de toma de decisiones y en los benéficos que se deriven de la utilización de los recursos genéticos, así como de las aplicaciones y comercializaciones subsiguientes de manera justa y equitativa (art. 5). Además, establece el deber de que el acceso a los conocimientos tradicionales asociados a los recursos genéticos que se encuentren en posesión de pueblos originarios o comunidades locales, se produzca a través de un proceso de consulta previa, libre e informada o con su consentimiento previo y participación efectiva (art. 7). En relación con los gobiernos de los Estados Parte, estos se obligan a tomar en consideración las leyes consuetudinarias, protocolos y procedimientos comunitarios con respecto a los conocimientos tradicionales asociados a recursos genéticos (art. 12.1) y los insta a apoyar a los pueblos originarios para que desarrollen sus propios protocolos comunitarios, en relación con sus conocimientos tradicionales, y sobre los mecanismos para su participación justa y equitativa en los beneficios que se deriven de la utilización de tales conocimientos (art. 12.3).

Cabe destacar, que es de gran relevancia para el diseño de los procesos de autorregulación de la actividad turística, el hecho de que el Protocolo abra la posibilidad al reconocimiento estatal de la regulación comunitaria sobre el acceso a sus conocimientos tradicionales y recursos genéticos. En este sentido, durante los Talleres de Diseño de Productos Turísticos de Base Comunitaria en la Comuna de Panguipulli, se constató la existencia de huertos familiares asociados a varias de las iniciativas turísticas y generalmente al cuidado de las mujeres, alguno de los cuales son parte de los activos con los que

31 A La línea definida por el Protocolo de Nagoya, acerca del resguardo de los conocimientos tradicionales de las comunidades locales y pueblos originarios asociados a los recursos biológicos presentes en sus territorios, así como la regulación sobre los derechos de acceso a éstos, se opone el Convenio UPOV 91 de la ONG The International Union for the Protection of New Varieties of Plants (UPOV). Este amenaza la conservación de semillas locales que se pierden debido al desuso, estimula el uso de transgénicos y la contaminación genética, y favorece la apropiación de variedades nacionales a través del "derecho de obtentor" (para reproducir o multiplicar una variedad con fines comerciales protegida por este derecho, es necesario disponer de la autorización del obtentor). Además permite a las empresas semilleras perseguir judicialmente a los agricultores y embargar y destruir sus cosechas. 
cuentan algunas iniciativas turísticas. Así, por ejemplo, en el sector de Pocura Alto, doña Dominga Catricura dentro de sus servicios y actividades turísticas tiene un huerto medicinal y vivero, donde cuenta con una gran variedad de plantas medicinales, nativas y no nativas, conservando una gran diversidad de semillas ${ }^{32}$. Se suma a esto, las actividades de guiado que se desarrollan entre la rica diversidad de flora y fauna que hay en la Comuna de Panguipulli y su abundancia de productos no madereros.

De esta forma, considerar el Protocolo dentro de una política de fomento del turismo y la agricultura familiar, representa un avance a la hora de favorecer la autorregulación comunitaria sobre los conocimientos tradicionales y propender a la participación justa en los beneficios que se reporten. Esto, como hemos venido sosteniendo, va en la línea del principio de autodeterminación y control interno de los asuntos propios de los pueblos indígenas y comunidades locales. En este sentido, un proceso de autocertificación y autorregulación comunitaria de la actividad turística, que busque minimizar los efectos negativos que podría tener dicha actividad sobre el patrimonio natural y cultural de las comunidades locales, así como en los conocimientos tradicionales asociados a los recursos genéticos dentro de sus territorios, debiera contemplar, entre otras cosas, la posibilidad o no de acceder a dichos recursos genéticos y conocimientos tradicionales asociados, en atención a sus propios patrones culturales e institucionales y, en caso de permitir el acceso, normar los modos de utilización de los mencionados conocimientos y recursos genéticos asociados, en base a criterios de participación justa y equitativa en los beneficios que se deriven de la utilización de tales conocimientos en el desarrollo turístico. Esto que resulta fundamental si se quiere desplegar un turismo con enfoque de derechos humanos, comunitario y con pertinencia cultural, toda vez que, el patrimonio natural, cultural y tradicional es un atractivo diferenciadordelturismoquedesarrollanlascomunidadeslocalesenlacomunadePanguipulli.

\section{Reflexión crítica sobre los procesos de autocertificación en el} TBC desde los aportes del derecho internacional de los derechos humanos

Como veíamos al revisar la Ley de Turismo, el Sistema de certificación de la calidad turística se comprende bien desde una perspectiva de relaciones jurídicas plurales. Así, la propia Ley reconoce (o asume) espacios de producción normativa autónoma a sujetos no-estatales. Sin embargo, la crítica principal que se le puede realizar al Sistema de certificación de la calidad turística, es que genera condiciones desiguales para los distintos tipos de operadores turísticos y desconoce de facto las particularidades del TBC y sus actores principales. En primer lugar, porque las normas a las que tienen que adecuarse estas iniciativas (para que después se evalúe su grado de cumplimiento respecto a las mismas) han sido diseñadas por y para un sector de la industria (mediana y gran empresa), por lo que no son estándares que las iniciativas comunitarias puedan

32 Véase Rivera (2014) y Catalán (2014). 
cumplir fácilmente, al ser ajenos a su realidad y condiciones materiales. $\mathrm{Y}$, en segundo lugar, porque genera un sistema que aspira a diferenciar simbólicamente entre aquellos que cuentan con el sello y aquellos que no. De esta forma, aquellos que cuentan con el sello de calidad acceden a ventajas competitivas que los benefician, ampliando aún más la brecha entre éstos y las iniciativas que no pueden acceder al Sistema. En este sentido, la esfera de lo jurídico es, al fin y al cabo, un aspecto más de la realidad social, y como tal, se encuentra inmersa en una cultura donde se establecen relaciones de poder generalmente desiguales. Desde un punto de vista conceptual, el origen del proceso normativo que estamos analizando, la normalización y certificación turística, es uno de sus elementos definitorios y característicos, para que este tipo de gestión de la actividad turística refleje las particularidades de los emprendimientos de TBC. Por ello, potenciar las iniciativas de las comunidades locales y pueblos indígenas para que desarrollen sus propios procesos de certificación (o autocertificación) resulta del todo apropiado con la realidad y condiciones económicas, sociales, culturales y territoriales de estos pueblos y comunidades. Esto supone una materialización concreta del ejercicio efectivo del derecho a la autodeterminación, el que ofrece una base mínima de elementos para generar un diálogo entre autoridades y comunidades, así como argumentos para el reconocimiento, fomento y promoción de dicho proceso, que va en la misma dirección de las actuales tendencias en el desarrollo de la actividad turística por parte de comunidades a nivel latinoamericano. No obstante, debido al incipiente estado de desarrollo en el que se encuentran los procesos de autorregulación comunitaria, el efecto en la mejora de las prácticas comunitarias del turismo está en permanente descubrimiento. Lo que sí es claro es que el desarrollo de los emprendimientos de base comunitaria es "muchas veces lento y con variados problemas y conflictos que obedecen a la naturaleza de trabajo colectivo ${ }^{33}$ ", , por lo que resulta indispensable contar con un fuerte compromiso y voluntad de las comunidades que lideran este tipo de iniciativas en la comuna. De esta manera, aunque es fundamental, tampoco basta con que la regulación y autocertificación del TBC surjan de deliberaciones internas. Se requiere además que todos los sujetos implicados en el universo de la actividad turística (re)conozcan la existencia y el valor de dichos instrumentos, lo que incluye tanto a las comunidades anfitrionas, como a los visitantes, y muy particularmente a los organismos públicos y privados.

33 En ese sentido, véanse las referencias a los Talleres de Diseño de productos de base comunitaria en Rivera (2014). 


\section{Referencias bibliográficas}

Anaya, J. (2005). Los pueblos indígenas en el derecho internacional. Madrid:Trotta. Aylwin, J. Meza-Lopehandía, M., Yáñez, N. (2014). Los Pueblos Indígenas y el Derecho. Santiago: LOM.

Aylwin, J., Cuadra, J. (2011). Los desafíos de la conservación en los territorios indígenas en Chile. Temuco: Observatorio de Derechos de los Pueblos Indígenas. Recuperado de: http://iccaconsortium.org/wp-content/uploads/images/stories/Database/ los_desafos_de_la_conservacion.pdf Aylwin, J. (2009). El derecho internacional de los derechos humanos y los pueblos indígenas. En M. Meza-Lopehandía (Ed.), Las implicancias de la ratificación del Convenio No 169 de la OIT en Chile, Temuco: Observatorio Ciudadano.

Biblioteca del Congreso Nacional (2010). Historia de la Ley No 20.423. Del sistema institucional para el desarrollo del Turismo.

Catalán, G. (2014). Estudio de casos sobre las experiencias y significaciones de mujeres mapuche y campesinas en la Feria Gastronómica y Artesanal de Pocura, comuna de Panguipulli Región de los Ríos. Tesis para la obtención del grado de Licenciada en Antropología, Universidad Austral de Chile. Recuperado de: http:// cybertesis.uach.cl/tesis/uach/2015/ffc357e/doc/ffc357e.pdf

Cover, R. (2002). Derecho, narración y violencia. Poder constructivo y poder destructivo en la interpretación judicial. Barcelona: Gedisa.

Guerra, F. (2014a). Territorios ancestrales, derechos humanos y concesiones administrativas en áreas silvestres protegidas del Estado. Una mirada desde una teoría jurídica crítica al desarrollo del turismo de base comunitaria. Trabajo presentado en el 1er Congreso de Antropología Jurídica de la Red Latinoamericana de Antropología Jurídica (RELAJU) de 2014, Santiago, Chile.

Guerra, F. (2014b). Hacia un proceso de autocertificación comunitaria de calidad de las iniciativas turísticas en el Destino Siete Lagos: Una perspectiva normativa global, regional, nacional y local (Informe). Valdivia: CEAM-UACh.

Guerra, F., Gómez M.L (col.) (2014). Análisis crítico de los modelos de gestión indirecta en áreas silvestres protegidas del Estado: Un estudio desde el Derecho Administrativo y el Derecho Internacional para la propuesta de un modelo de cogestión en el contexto del desarrollo turístico comunitario (Informe). Valdivia: CEAM-UACh.

Henríquez, C., Pilquimán, M., Pino, A. (2013). Turismo Comunitario: rescate cultural, conservación de la biodiversidad y desarrollo de base local. Trabajo presentado en el X Congreso Latinoamericano de Reservas Privadas y Pueblos Originarios de 2013, Valdivia, Chile. Recuperado de: http://asiconservachile.cl/fileadmin/templates/data_users/X_CONGRESO_2013/ Presentaciones/Turismo/Cristian_Henriquez.pdf 
Maldonado, C. (2006). Turismo y comunidades indígenas: Impactos, pautas para la autoevaluación y códigos de conducta. Ginebra: Organización Internacional del TrabajoPrograma de Desarrollo de Pequeñas Empresas, Documento de trabajo No 79, Serie Red de Turismo Sostenible Comunitario para América Latina (REDTURS). Recuperado de: http://www.ilo.org/wcmsp5/groups/public/@ed_emp/@emp_ent/@ifp_seed/documents/ publication/wcms_117521.pdf

Morales, H. (2006). Turismo comunitario: una nueva alternativa de desarrollo indígena, en AIBR. Revista de Antropología Iberoamericana, 1(2), 249-264. Recuperado de: http://www.redalyc.org/articulo.oa?id=62310205

Oficina Internacional del Trabajo (2009). Informe de la Comisión de Expertos en Aplicación de Convenios y Recomendaciones (Conferencia Internacional del Trabajo, 98.a reunión). Ginebra: Oficina Internacional del Trabajo. Recuperado de: http://www. ilo.org/wcmsp5/groups/public/@ed_norm/@relconf/documents/meetingdocument/ wcms_103488.pdf

----- (2006). Memoria del IV Encuentro Latinoamericano, Códigos de conducta y uso de marcas, realizado en Panamá en 2005. Ginebra: Oficina Internacional del Trabajo, Documento de trabajo $\mathrm{N}^{\mathrm{o}} 77$, Serie Red de Turismo Sostenible Comunitario para América Latina (REDTURS). Recuperado de: http://www.ilo.org/wcmsp5/groups/ public/@ed_emp/@emp_ent/@ifp_seed/documents/publication/wcms_117523.pdf Pacheco, G., Henríquez, C., Sampaio, C., Oyarzún, E. (2011). Encadenamientos socioproductivos y ecosocioeconomía de las organizaciones. Análisis propositivo para el Turismo de Intereses Especiales (TIE) en el territorio lacustre de la región de la Araucanía. En Gestión Turística, 16, 49-68. Recuperado de: DOI:10.4206/gest.tur.2011. n16-03

Pereiro, X. (2014). Antropología del turismo indígena en Gula Yala (Panamá). Tesis doctoral, Universidad de La Laguna. Recuperado de: http://hdl.handle.net/10348/2934 Rivera, F. (2014). Informe del Proyecto Transferencia Tecnológica, Turismo de Intereses Especiales (TIE) y Desarrollo con Base Local en el Destino Siete Lagos (Innova 13PDTR 23946), Talleres de Diseño de Productos Turísticos de base Comunitaria. Valdivia: CEAM-UACh.

Santos, B. De S. (2009). Sociología Jurídica Crítica. Para un nuevo sentido común en el derecho. Madrid: Trotta.

Skewes, J. C., Henriquez, C., y Pilquimán, M. (2012). Turismo comunitario o de base comunitaria: Una experiencia alternativa de hospitalidad vivida en el mundo mapuche. Tralcao sur de Chile. En Cultur, Revista de Cultura e Turismo, 6(2). Recuperado de: www.uesc.br/revistas/culturaeturismo

Stavenhagen, R. (2002). Derecho internacional y derechos indígenas. En E. Krotz (Ed.), Antropología jurídica: perspectivas socioculturales para el estudio del derecho, Barcelona:Anthropos/Universidad Autónoma Metropolitana-Iztapalapa. 
Yáñez, N. (2006). Reconocimiento legislativo de los derechos ambientales indígenas en el ámbito internacional. En M. Berraomdo (Coord.), Pueblos Indígenas y Derechos Humanos. Bilbao: Universidad de Deusto.

\section{Jurisprudencia}

Corte de Apelaciones de Valdivia (2012). Sentencia de 4 de mayo de 2012, en autos caratulado: Millaray Huichalaf Pradines y Otros C/ Juan H. Ortiz Ortiz, Rol 501-2011. Corte Interamericana de Derechos Humanos (2007). Caso del Pueblo Saramaka vs. Surinam. Sentencia del 28 de noviembre de 2007 (Excepciones Preliminares, Fondo, Reparaciones y Costas). Recuperado en: http://www.corteidh.or.cr/docs/casos/articulos/ seriec_172_esp.pdf

Tribunal Constitucional de Chile (2014). Sentencia de 6 de mayo de 2014, en autos sobre requerimiento de inaplicabilidad por inconstitucionalidad presentado por Enrique Eichin Zambrano respecto del artículo $5^{\circ}$, numerales $1^{\circ}$, inciso primero, y $3^{\circ}$ del Código de Justicia Militar, en los autos RIT 11463-2013, RUC N ${ }^{\circ}$ 1310018169-4, del Séptimo Juzgado de Garantía de Santiago, Rol 2493-13.

----- (2013). Sentencia de 13 de octubre de 2013, en autos sobre Requerimiento de inaplicabilidad por inconstitucionalidad presentado por la Universidad de los Andes respecto del artículo único de la Ley $\mathrm{N}^{\circ} 20.411$, en los autos sobre recurso de reclamación, caratulados "Universidad de Los Andes con Dirección General de Aguas", Rol ingreso Corte $\mathrm{N}^{\circ}$ 921-2012, de la Corte de Apelaciones de Santiago, Rol 2430-13.

----- (2008). Sentencia de 3 de abril de 2008, en autos sobre Control de constitucionalidad del proyecto de acuerdo aprobatorio relativo al Convenio $\mathrm{N}^{\mathrm{o}} 169$ sobre pueblos indígenas, adoptado por la Organización Internacional del Trabajo, de 27 de junio de 1989 (Boletín N²33-10), Rol 1050-08. 